\title{
Flohplagen - Wenn Katzenflöhe den Menschen heimsuchen
}

\author{
Flea Trouble - When Cat Fleas Plague the Human Being
}

Autoren

Institut
E. Rowe, G. Kolde, H. Meffert

Dermatologisches Zentrum Berlin
Bibliografie

DOI http://dx.doi.org/

10.1055/s-0042-100565

Online-Publikation: 29.3.2016

Akt Dermatol 2016; 42: 283-285

(c) Georg Thieme Verlag KG

Stuttgart · New York

ISSN 0340-2541

\section{Korrespondenzadresse}

Dr. med. Elisabeth Rowe

Dermatologisches Zentrum

Potsdamer Chaussee 80

14129 Berlin

rowe.elisabeth@t-online.de www.dermatologie-berlin.de

\section{Zusammenfassung \\ $\nabla$}

Bericht über das Auftreten zunächst unscheinbarer, kleiner Papeln, dann von mit quälendem Juckreiz und Schmerz begleiteten, teils aufgekratzten großen, prallen Blasen bei einer 51-jährigen Frau. Die Diagnose konnte erst gestellt werden, als die Patientin die Frage nach einer eigenen, frei laufenden Katze bejaht hatte.

In der Wohnung der Betroffenen wurden massenhaft Katzenflöhe (Ctenocephalides felis) gefunden. Es wird über den Flohnachweis in der häuslichen Umgebung der Patientin, die Differenzialdiagnosen, die Therapie und die Sanierung der Wohnung berichtet.

\section{Einführung \\ $\nabla$}

In Europa sind bis zu $80 \%$ der geschätzt 12 Millionen heimischen Hauskatzen vom etwa 1-3mm langen Katzenfloh Ctenocephalides felis ( $\bullet$ Abb.1) befallen [1].

Der 1-3mm lange Katzenfloh ist weltweit verbreitet. Er akzeptiert auch Mensch und Hund als Zwischenwirte. Auf menschlicher Haut entstehen nach den Stichen juckende, punktförmige, als Flohstraßen angeordnete Rötungen mit hellem Hof, gelegentlich auch kleine Papeln. In letzter Zeit konsultierten uns mehrere Patienten mit großen und prallen, teils aufgekratzten, von quälendem Juckreiz, gelegentlich sogar von Schmerz begleiteten Blasen. Dabei handelt es sich möglicherweise um allergische Reaktionen vom Spättyp. Wenn Arzt und Patient nicht an Katzen als mögliche Überträger von Flöhen denken, kann die Diagnose oft nur spät oder nicht gestellt werden.

\section{Anamnese \\ $\nabla$}

Die 51-jährige Frau wird seit Jahren wegen atopischen Ekzems und Rhinitis allergica behandelt.

8. 10.2014: An Hals und Beinen bestehen multiple, mit Krusten belegte Exkoriationen. Deren Umgebung ist gering angeschwollen und juckt stark. Auch mittels Lupe ist kein Eiter erkennbar. Keine Skabies.

Die Frau vermutet als Ursache familiären Stress. Diagnose: unklar. Therapie: Telfast $120 \mathrm{mg}$ FTA N1; DAC Basiscreme 100,0.

16.10.2014: Subjektiv etwas besser. Diagnose weiterhin unklar. Große Blasen auf beiden Fußrücken. Therapie: hydrophile Chlorhexidingluconat-Creme 1\% (NRF 11.116.) ( $\bullet$ Abb.2, $\bullet$ Abb.3). 17. 10.2014: Neue Blasen. Patientin: „Es juckt und juckt.“

Labor (auszugsweise): IgE 519U/ml (normal bis zu $100 \mathrm{U} / \mathrm{ml}$ ), bekannte leichte hypochrome Anämie, keine Porphyrie. Diagnostische Exzision in Erwägung gezogen.

07.11.2014: während des Urlaubs auf Usedom völlige Abheilung. Infolge von Meerwasser und UV-Strahlen? Rezidiv nach Waldspaziergängen in Brandenburg. Eichenprozessionsspinner? Aber Hauterscheinungen nur noch an den Beinen, nicht mehr am Hals. Aktuell diagnostische Exzision nicht indiziert, weil derzeit lediglich Erosionen mit Blasenresten bestehen, keine frischen Blasen.

Vorläufige Diagnose: Insektenstiche, Grasmilben? Aus klinischer Sicht ist das Krankheitsbild offensichtlich exogen ausgelöst. Der erhöhte IgE-Spiegel, der Befall vom Fuß bis zur Unterschenkelmitte und das Abheilen während des Strandurlaubs auf Usedom sprechen für eine exogen-allergische Induktion. Differenzierung des IgE in einem Allergiezentrum erwogen.

11.11.2014: Neue flache Quaddeln an Oberund Unterschenkeln, maximal fingernagelgroß, derb, vereinzelt exkoriiert, nicht aber bullös. 


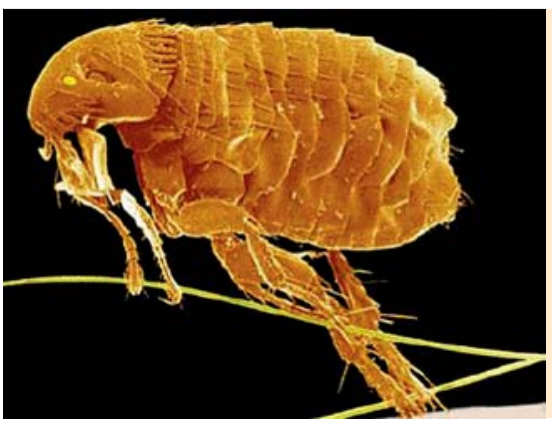

Abb. 1 Rasterelektronenmikroskopisches Bild eines Katzenflohs.

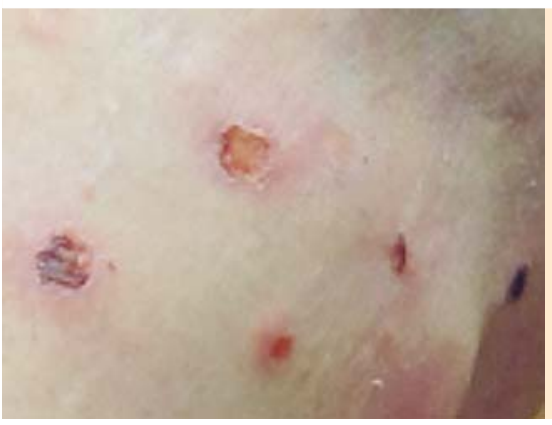

Abb. 2 Mit Krusten belegte Exkoriationen. Die Umgebung ist gering angeschwollen und juckt stark.
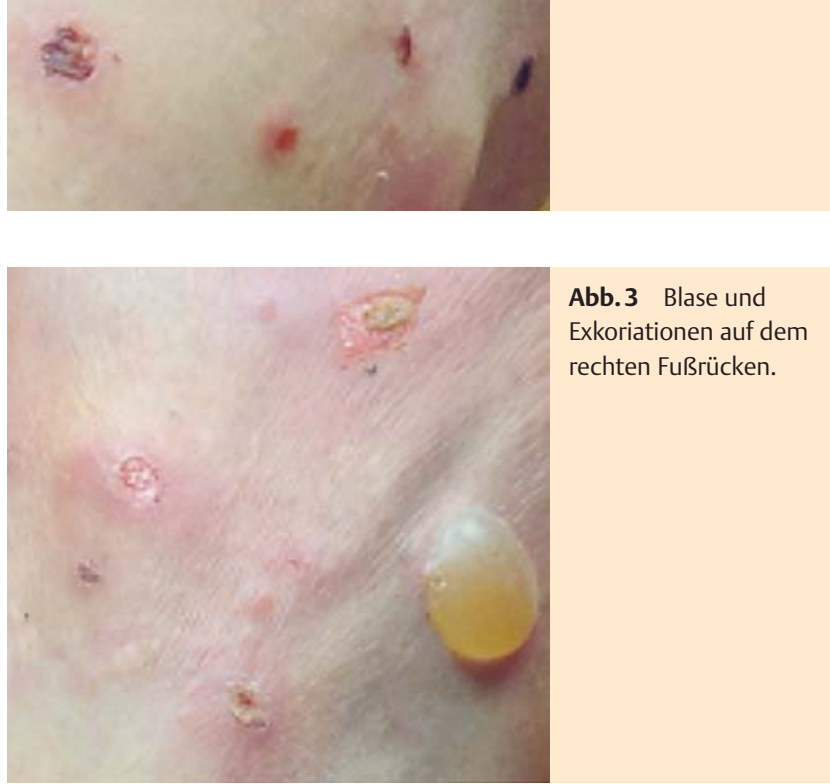

Abb. 3 Blase und Exkoriationen auf dem rechten Fußrücken.

Therapie: Decortin H, 50-mg-Tabletten, eine täglich über zunächst sieben Tage.

18.11.2014: Urtikarielle Dermatitis unter $50 \mathrm{mg}$ Decortin $\mathrm{H}$ abgeheilt. Kortison abgesetzt.

22.11.2014: Alte Stellen heilen ab, neue kommen hinzu. Auf Katzen angesprochen: „Ja, Freigänger, mal tagelang weg, bis zu zwei oder drei Wochen." Die Töchter hätten inzwischen auch solche juckenden Stellen, nur nicht so schlimm. Flohbekämpfung wird betrieben ( siehe unter „Sanierung der Wohnung“). An Flöhe habe sie auch schon gedacht, sich aber geschämt. Die Frau ist über die Diagnose Flohstiche erleichtert. Nun muss sie die Viecher bekämpfen und die Grübelei, woher das denn komme, hat ein Ende.

27. 11. 14: Flohsanierung läuft, aber es ist schwierig - Haus, separate Wohnung, Teppiche, Brücken und ein Mann, der die gerade entseuchten Brücken wieder auf den noch verkeimten Teppich legt. Fragt nach Fenistil-Spritze - abgelehnt. Tabletten wirken ebenso.

20.05.2015: Patientin berichtet, dass die Freigänger-Katze vor einiger Zeit überfahren worden sei. Dann habe die Flohplage irgendwann ein Ende gefunden.

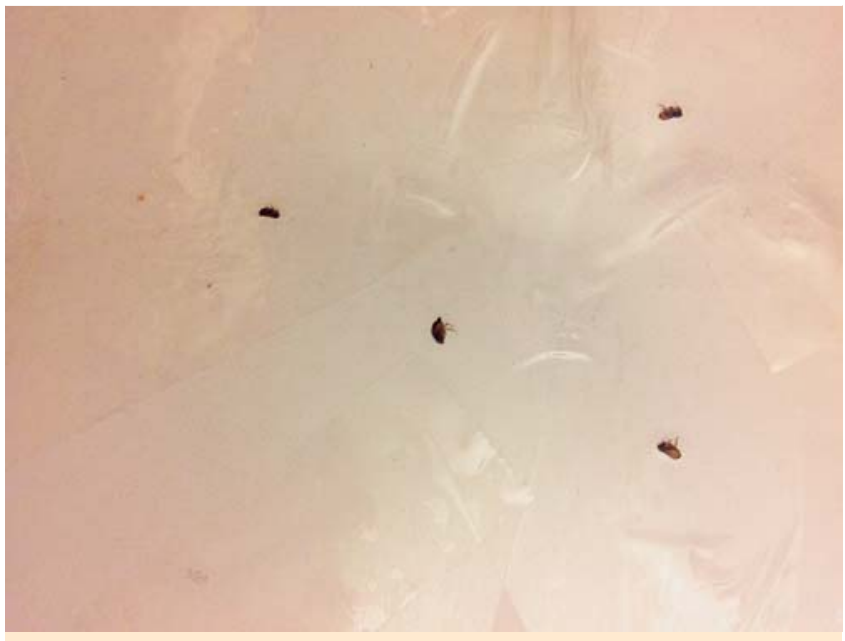

Abb.4 Von der Patientin mittels Tesafilm eingefangene Katzenflöhe.

\section{Flohnachweis \\ $\nabla$}

Weibliche Flöhe legen ihre Eier im Fell der sie beherbergenden Katze ab. Die Eier fallen dann zu Boden und sind deshalb vor allem dort zu finden, wo sich die Katze häufig aufhält. Über ein Larven- und ein Puppenstadium entwickeln sich adulte Flöhe, die mit bloßem Auge erkennbar sind ( $\bullet$ Abb.4).

Der Befall von Katze respektive Wohnung kann auch über den Flohkot erkannt werden. Im Flohkot sind Abbauprodukte des Blutfarbstoffs enthalten. Diese geben sich als rötliche Flecke, oder im Wood-Licht als ziegelrote Fluoreszenz, zu erkennen. Hierzu wird ein gut angefeuchtetes Blatt weißes Papier oder weißes Handtuch unter und neben die Katze gelegt. Dann fährt man mit einer Bürste oder mit der Hand durch deren Rückenfell. Es fallen Haare, Schuppen, Schmutz und ggf. auch Flohkot auf die feuchte Unterlage. Anders als Schmutz gibt der Flohkot in feuchtes Milieu rötliche Farbstoffe ab.

\section{Therapie}

$\nabla$

Empfohlen werden Lotio alba mit oder ohne Zusatz von 2-4\% Polidocanol, erforderlichenfalls auch kurzfristig topische Kortikoide, bei starkem Juckreiz Antihistaminika wie Dimetinden (z.B. Fenistil ${ }^{\circledR}$-Dragees) [2].

Die Behandlung der Katze überlässt man dem Tierarzt, den man besser nach aktuellen Flohmitteln zur Sanierung der Wohnung fragen sollte. Solche Mittel sind übrigens nicht in der Apotheke, sondern nur direkt beim Tierarzt zu bekommen.

\section{Sanierung der Wohnung}

Die Bekämpfung der Flöhe erwies sich als weitaus aufwendiger als angenommen. Während die adulten Flöhe Blut saugen, ernähren sich die Larven von allerlei organischen Partikeln und dem Kot ihrer Vorfahren. Derartiges finden sie vor allem in Ritzen des Fußbodens, im Teppichboden, in Treppenstufen mit Textilbelag u.ä., und besonders an Schlafstellen von Mensch und Katze. Zur Sanierung der Räume, zu der die beschuldigte Katze Zugang hatte, und insbesondere der genannten Lagerstätten und deren Umgebung wurden Antiparasitika mit den Wirkstoffen Perme- 
thrin und Pyriproxyfen mittels Spray und automatischem Vernebler angewandt.

Erforderlich sind zudem

- wiederholtes Staubsaugen der Teppiche, Teppichböden und Polstermöbel

- wiederholtes Wischen der Böden und Fußleisten mit Syndets enthaltendem Wischwasser

- Waschen der von der Katze kontaktierten Textilien, möglichst bei $65^{\circ} \mathrm{C}$. Die Temperatur ist nicht so wichtig. Wichtig ist die Verwendung von Syndets, da durch diese die Atemwege der Flöhe gestört werden. So gesehen reicht auch das Waschen und Wischen mit kaltem Wasser.

\section{Interessenkonflikt}

$\nabla$

Die Autoren geben an, dass keine Interessenkonflikte bestehen.

\section{Abstract}

\section{Flea Trouble - When Cat Fleas Plague the Human Being} $\nabla$

Report on the appearance of itching but initially unimpressive small papules, than of large and tight, partially excoriated bladders which were accompanied by heavy pruritus and pain in a 51-year-old woman.

Not till the patient approved the question whether she has her own but free running cat, the infestation with cat fleas was diagnosed. Large amounts of the cat flea Ctenocephalides felis were found at the flat of the woman.

It is reported on the detection of fleas at her domestic environment, on differential diagnostics, on treatment of the patient, and on cleaning up the flat.

\section{Literatur}

1 Voigt TF. Hunde und Katzen vor Parasiten schützen. Pharmazeutische Zeitung online; 19/2006

2 Braun-Falco O, Plewig G, Wolff HH. Dermatologie und Venerologie. Berlin, Heidelberg, New York: Springer-Verlag; 1996: 323-325 